IJMMS 31:2 (2002) 77-84

PII. S016117120210620X

http://ijmms.hindawi.com

(c) Hindawi Publishing Corp.

\title{
BORN-INFELD ELECTRODYNAMICS: CLIFFORD NUMBER AND SPINOR REPRESENTATIONS
}

\author{
ALEXANDER A. CHERNITSKII
}

Received 25 June 2001 and in revised form 26 December 2001

\begin{abstract}
The Clifford number formalism for Maxwell equations is considered. The Clifford imaginary unit for space-time is introduced as coordinate independent form of fully antisymmetric fourth-rank tensor. The representation of Maxwell equations in massless Dirac equation form is considered; we also consider two approaches to the invariance of Dirac equation with respect to the Lorentz transformations. According to the first approach, the unknown column is invariant and according to the second approach it has the transformation properties known as spinorial ones. The Clifford number representation for nonlinear electrodynamics equations is obtained. From this representation, we obtain the nonlinear like Dirac equation which is the form of nonlinear electrodynamics equations. As a special case we have the appropriate representations for Born-Infeld nonlinear electrodynamics.
\end{abstract}

2000 Mathematics Subject Classification: 35Q60, 15A66.

1. Introduction. At present Born-Infeld nonlinear electrodynamics arouse considerable interest for various points of view. There are some propositions which allow considering this nonlinear electrodynamics as a unique field model. This model, considered by Born and Infeld [1], is obtained from some variational principle proposed by Eddington [9]. This model appears also in the string theory [11]. It was shown in [2] that the electrodynamical part of the Einstein unified field model, with nonsymmetrical metric [10], is equivalent to Born-Infeld electrodynamics. The characteristic equation for Born-Infeld electrodynamics has a very notable form [3]. Also, some propositions which set off Born-Infeld electrodynamics from electrodynamical models, are presented in [7]. About author's opinion on a significance of such nonlinear electrodynamics models, see [5].

At present, Born-Infeld model is considered as a possible vacuum electrodynamics and future experiments must have solved what model is more appropriate [6].

Thus, any results which may help us in the mathematical investigation of this field model are strongly welcomed.

For the conventional Maxwell electrodynamics we know the Clifford number representation (see [12, 14]). Maxwell system of equations may be written in highly compact form for this representation. In addition, it may be more convenient for some mathematical problems. Clifford numbers give also the representation of Maxwell system in massless Dirac equation form, that may help to find more profound connections between electrodynamics and quantum mechanics.

In this connection, the application of Clifford numbers for representation of BornInfeld nonlinear electrodynamics model is the important problem. 
2. Clifford numbers and Maxwell equations. Clifford numbers are convenient enough for the treatment of geometric objects in space-time. In particular, we can relate the tensor of electromagnetic field to the Clifford number by the formula

$$
\hat{F}=\frac{1}{2} F_{\mu \nu} \hat{\gamma}^{\mu} \hat{\gamma}^{v}=E_{i}\left(\hat{\gamma}^{i} \wedge \hat{\gamma}^{0}\right)+\frac{1}{2} \varepsilon_{0 i j k} B^{k} \hat{\gamma}^{i} \hat{\gamma}^{j},
$$

where the Greek indexes take the values $0,1,2,3$, the Latin ones take the values 1,2 , 3 , and $\varepsilon_{\mu v \sigma \rho}$ are the components of fully antisymmetric fourth-rank tensor such that $\varepsilon_{0123}=\sqrt{|g|}$. Here we use the designations for electromagnetic field vectors which is appropriate to arbitrary coordinate system [4]. We designate the Clifford numbers by letters with hat, as distinct from ordinary numbers.

Symmetric (inner) and antisymmetric (exterior) Clifford number products are designated by a dot $(\cdot)$ and a wedge $(\wedge)$ accordingly. For the reference vectors $\hat{\gamma}^{\mu}$ we have $\hat{\gamma}^{\mu} \cdot \hat{\gamma}^{v}=\hat{1} g^{\mu \nu}$. They can be represented by Dirac's matrices and $\hat{1}$ means unit Clifford number or $(4 \times 4)$ unit matrix.

Notice that $\hat{F}$ in (2.1) is an invariant geometrical object. This means, in particular, that in the matrix representation the appropriate matrix components do not depend on used coordinate system.

If we introduce the invariant differential operator

$$
\hat{\partial} \equiv \hat{\gamma}^{\mu} \frac{\partial}{\partial x^{\mu}} \equiv \hat{\gamma}^{\mu} \partial_{\mu}
$$

then the equation

$$
\hat{\partial} \hat{F}=0
$$

is a coordinate-free form of Maxwell system of equations (without currents). For the specified form of Dirac's matrices, we can easily obtain the appropriate coordinate representation from (2.3).

We introduce some designations concerning Clifford numbers, which we will use:

$$
\begin{gathered}
\hat{\rho}^{i} \equiv \hat{\gamma}^{i} \wedge \hat{\gamma}^{0} \Longrightarrow \hat{\rho}^{i} \cdot \hat{\rho}^{j}=-g^{00} g^{i j}+g^{i 0} g^{j 0}, \\
\hat{\rho}^{i} \cdot \hat{\rho}_{j} \equiv \delta_{j}^{i} \Longrightarrow \hat{\rho}_{i}=\hat{\gamma}_{0} \wedge \hat{\gamma}_{i},
\end{gathered}
$$

where $\hat{\gamma}^{\mu} \cdot \hat{\gamma}_{v} \equiv \hat{1} \delta_{v}^{\mu}$, and

implies

$$
\hat{\imath} \equiv \frac{1}{4 !} \varepsilon_{\mu \nu \sigma \rho} \hat{\gamma}^{\mu} \hat{\gamma}^{v} \hat{\gamma}^{\sigma} \hat{\gamma}^{\rho}
$$

$$
(\hat{\imath})^{2}=-\hat{1}, \quad \hat{\gamma}^{j} \hat{\imath}=-\hat{\imath} \hat{\gamma}^{j}, \quad \hat{\rho}^{j} \hat{\imath}=\hat{\imath} \hat{\rho}^{j} .
$$

As we see, the Clifford number $\hat{\imath}$ is coordinate-free form of fully antisymmetric fourth-rank tensor. Because for space-time $\hat{\imath}^{2}=-\hat{1}$, we can call it here Clifford imaginary unit. (This appellation is not allowable for arbitrary space because there are spaces for which construction of type (2.5) has unit square.)

By analogy with ordinary complex numbers, we introduce the conjugation operation for Clifford numbers in four-dimensional space-time. Any such Clifford number or any $(4 \times 4)$ real matrix can be represented as

$$
\begin{aligned}
\hat{C} & =a \hat{1}+b_{\mu} \hat{\gamma}^{\mu}+c_{i} \hat{\rho}^{i}+d_{i} \hat{\imath} \hat{\rho}^{i}+e_{\mu} \hat{\imath} \hat{\gamma}^{\mu}+f \hat{\imath} \\
& =(a+\hat{\imath} f) \hat{1}+\left(b_{\mu}+\hat{\imath} e_{\mu}\right) \hat{\gamma}^{\mu}+\left(c_{i}+\hat{\imath} d_{i}\right) \hat{\rho}^{i},
\end{aligned}
$$


where $a, b_{\mu}, c_{i}, d_{i}, e_{\mu}, f$ are real numbers, and we define the conjugate Clifford number as

$$
\begin{aligned}
\hat{C}^{\dagger} & =(a-\hat{\imath} f) \hat{1}+\left(b_{\mu}-\hat{\imath} e_{\mu}\right) \hat{\gamma}^{\mu}+\left(c_{i}-\hat{\imath} d_{i}\right) \hat{\rho}^{i} \\
& =a \hat{\imath}+b_{\mu} \hat{\gamma}^{\mu}+c_{i} \hat{\rho}^{i}-d_{i} \hat{\imath} \hat{\rho}^{i}-e_{\mu} \hat{\imath} \hat{\gamma}^{\mu}-f \hat{\imath} .
\end{aligned}
$$

Using (2.4), (2.5), (2.6), (2.7), and (2.8) we can write

$$
\hat{F}=\hat{E}-\hat{\imath} \hat{B}, \quad \hat{F}^{\dagger}=\hat{E}+\hat{\imath} \hat{B},
$$

where

$$
\hat{E} \equiv E_{i} \hat{\rho}^{i}, \quad \hat{B} \equiv B^{i} \hat{\rho}_{i} .
$$

The Clifford number (2.7) is an invariant geometrical object and we can mark out three parts of it. In the representation $(2.7 \mathrm{~b})$ the first part is scalar because the coefficients $a$ and $f$ are scalars for arbitrary coordinate transformation. The second part is vector and the coefficients $b_{\mu}$ and $e_{\mu}$ transform like vector's components. The third part is known as bivector and, as we see, it is an antisymmetrical second-rank tensor.

In (2.7) we call $a$ the real part of scalar and $\hat{\imath} f$ the imaginary part of it. Also, we mark out real $\left(b_{\mu} \hat{\gamma}^{\mu}\right)$ and imaginary $\left(\hat{\imath} e_{\mu} \hat{\gamma}^{\mu}\right)$ parts of vector, and real $\left(c_{i} \hat{\rho}^{i}\right)$ and imaginary $\left(\hat{\imath} d_{i} \hat{\rho}^{i}\right)$ parts of bivector.

Equation (2.3) is written also in the form

$$
\hat{\partial} \cdot \hat{F}+\hat{\partial} \wedge \hat{F}=0
$$

Using representation (2.9), we can write it in the following form:

$$
\hat{\gamma}^{0} \partial_{0} \wedge \hat{E}+\hat{\gamma}^{i} \partial_{i} \cdot \hat{E}+\hat{\gamma}^{i} \partial_{i} \wedge \hat{E}-\hat{\gamma}^{0} \partial_{0} \cdot \hat{\imath} \hat{B}-\hat{\gamma}^{i} \partial_{i} \cdot \hat{\imath} \hat{B}-\hat{\gamma}^{i} \partial_{i} \wedge \hat{\imath} \hat{B}=0
$$

Here we use that $\hat{\gamma}^{0} \cdot \hat{\rho}_{i}=0, \hat{\gamma}^{0} \wedge \hat{\imath} \hat{\rho}_{i}=0$ and for simplicity we consider that the reference vectors $\hat{\gamma}^{\mu}$ do not depend on coordinates. The left-hand side of (2.12) is a vector. We single out its time and space components, real and imaginary parts in each of them. As a result, we have the system

$$
\begin{gathered}
\hat{\gamma}^{i} \partial_{i} \wedge \hat{E}=0, \\
\hat{\gamma}^{i} \partial_{i} \cdot \hat{\imath} \hat{B}=0, \\
\hat{\gamma}^{0} \partial_{0} \wedge \hat{E}-\hat{\gamma}^{i} \partial_{i} \wedge \hat{\imath} \hat{B}=0, \\
\hat{\gamma}^{0} \partial_{0} \cdot \hat{\imath} \hat{B}-\hat{\gamma}^{i} \partial_{i} \cdot \hat{E}=0 .
\end{gathered}
$$

Here we use (2.10) and the following relations:

$$
\begin{array}{lll}
\hat{\gamma}^{i} \wedge \hat{\rho}_{j}=-\delta_{j}^{i} \hat{\gamma}_{0}, & \hat{\gamma}^{0} \wedge \hat{\rho}_{i}=\hat{\gamma}_{i}, & \hat{\gamma}^{i} \wedge \hat{\imath} \hat{\rho}^{j}=-\varepsilon^{0 i j k} \hat{\gamma}_{k}, \\
\hat{\gamma}^{i} \cdot \hat{\imath} \hat{\rho}_{j}=\delta_{j}^{i} \hat{\imath} \hat{\gamma}_{0}, & \hat{\gamma}^{0} \cdot \hat{\imath} \hat{\rho}_{i}=-\hat{\imath} \hat{\gamma}_{i}, & \hat{\gamma}^{i} \cdot \hat{\rho}^{j}=-\varepsilon^{0 i j k} \hat{\imath} \hat{\gamma}_{k},
\end{array}
$$

where $\varepsilon^{0123}=-1 / \sqrt{|g|}$. 
We can easily verify that system (2.13) is Maxwell system of equations, where the left-hand sides of (2.13a) and (2.13b) are divergences of electromagnetic field vectors.

Now, take a Cartesian coordinate system with $-g^{00}=g^{11}=g^{22}=g^{33}=1$ and $g^{\mu \nu}=0$ for $\mu \neq \nu$. For this case $\hat{\imath}=\hat{\gamma}^{0} \hat{\gamma}^{1} \hat{\gamma}^{2} \hat{\gamma}^{3}$.

Consider the appropriate Dirac's matrices in the form

$$
\begin{array}{rlrl}
\hat{\gamma}^{0} & =\left(\begin{array}{cccc}
i & 0 & 0 & 0 \\
0 & i & 0 & 0 \\
0 & 0 & -i & 0 \\
0 & 0 & 0 & -i
\end{array}\right), & \hat{\gamma}^{1}=\left(\begin{array}{cccc}
0 & 0 & 0 & i \\
0 & 0 & i & 0 \\
0 & -i & 0 & 0 \\
-i & 0 & 0 & 0
\end{array}\right), \\
\hat{\gamma}^{2}=\left(\begin{array}{cccc}
0 & 0 & 0 & -1 \\
0 & 0 & 1 & 0 \\
0 & 1 & 0 & 0 \\
-1 & 0 & 0 & 0
\end{array}\right), & \hat{\gamma}^{3}=\left(\begin{array}{cccc}
0 & 0 & i & 0 \\
0 & 0 & 0 & -i \\
-i & 0 & 0 & 0 \\
0 & i & 0 & 0
\end{array}\right) .
\end{array}
$$

As we can see, the matrix $\hat{F}$ in (2.1) has four independent complex components only. We designate the first column of the matrix $\hat{F}$ by $\bar{F}$, then

$$
\bar{F}=\hat{F} \overline{1}=\left(\begin{array}{c}
\bar{F}_{1} \\
\bar{F}_{2} \\
\bar{F}_{3} \\
\bar{F}_{4}
\end{array}\right)=\left(\begin{array}{c}
-i B_{3} \\
-i B_{1}-B_{2} \\
E_{3} \\
E_{1}-i E_{2}
\end{array}\right), \quad \overline{1} \equiv\left(\begin{array}{l}
1 \\
0 \\
0 \\
0
\end{array}\right) .
$$

And the matrices $\hat{F}$ and $\hat{F}^{\dagger}$ can be written in the following form:

$$
\begin{aligned}
\hat{F} & =\left(\begin{array}{cccc}
\bar{F}_{1} & -\bar{F}_{2}^{*} & \bar{F}_{3} & \bar{F}_{4}^{*} \\
\bar{F}_{2} & \bar{F}_{1}^{*} & \bar{F}_{4} & -\bar{F}_{3}^{*} \\
\bar{F}_{3} & \bar{F}_{4}^{*} & \bar{F}_{1} & -\bar{F}_{2}^{*} \\
\bar{F}_{4} & -\bar{F}_{3}^{*} & \bar{F}_{2} & \bar{F}_{1}^{*}
\end{array}\right), \\
\hat{F}^{\dagger} & =\left(\begin{array}{ccccc}
-\bar{F}_{1} & \bar{F}_{2}^{*} & \bar{F}_{3} & \bar{F}_{4}^{*} \\
-\bar{F}_{2} & -\bar{F}_{1}^{*} & \bar{F}_{4} & -\bar{F}_{3}^{*} \\
\bar{F}_{3} & \bar{F}_{4}^{*} & -\bar{F}_{1} & \bar{F}_{2}^{*} \\
\bar{F}_{4} & -\bar{F}_{3}^{*} & -\bar{F}_{2} & -\bar{F}_{1}^{*}
\end{array}\right) .
\end{aligned}
$$

Extracting the first column in the matrix equation (2.3), we obtain the following representation of Maxwell equations in the form of massless Dirac equation:

$$
\hat{\partial} \bar{F}=\hat{\gamma}^{\mu} \frac{\partial \bar{F}}{\partial x^{\mu}}=0 .
$$

Note that system (2.13) is an overdetermined one, because there are eight equations for six unknown functions $E_{i}(x), B_{i}(x)$. Traditionally, the determined system (2.13c), (2.13d) with additional conditions (2.13a) and (2.13b) are considered. These conditions are conserved in time according to (2.13c) and (2.13d). Thus, if we have the problem with initial conditions which satisfy (2.13a) and (2.13b), then (2.13a) and (2.13b) will be satisfied for any point of time. But general solution of system (2.13c) and (2.13d) is not general solution of Maxwell equations. Formally, system (2.18) has four complex 
equations for four complex unknown functions or column $\bar{F}(x)$ in (2.16). But in this case, we have also two additional conditions that $\bar{F}_{1}$ is pure imaginary and $\bar{F}_{3}$ is real. Of course, the form of such conditions is connected with the specified form of the matrix representation for $\hat{\gamma}^{\mu}$ which we use.

3. Coordinate transformations and spinors. Because we have Maxwell equations in massless Dirac equation form (2.18), it is necessary to elucidate the transformation properties of an unknown column $\bar{F}$. As noted above, because $\hat{\gamma}^{\mu}$ are the reference vectors, the operator $\hat{\partial}$ and the bivector $\hat{F}$ are invariant geometrical objects. Thus, if we have two coordinate systems $\left\{x^{\mu}\right\}$ and $\left\{x^{\prime \mu}\right\}$, then

$$
\hat{\partial}=\hat{\gamma}^{\mu} \frac{\partial}{\partial x^{\mu}}=\hat{\gamma}^{\prime} \frac{\partial}{\partial x^{\prime} v}, \quad \hat{F}=F_{\mu \nu} \hat{\gamma}^{\mu} \hat{\gamma}^{v}=F_{\mu \nu}^{\prime} \hat{\gamma}^{\prime \mu} \hat{\gamma}^{\prime} v .
$$

Consider the Lorentz transformation

$$
x^{\prime v}=L_{. \mu}^{v} x^{\mu}
$$

Then for Dirac's matrices we have

$$
\hat{\gamma}^{\prime} v=L_{. \mu}^{v} \hat{\gamma}^{\mu}=\hat{\Lambda}^{-1} \hat{\gamma}^{v} \hat{\Lambda}
$$

where $\hat{\Lambda}$ is some matrix.

When we relate a specified form of Dirac's matrices (e.g., (2.15)) with appointed coordinate system $\left\{x^{\mu}\right\}$, then we define invariant matrix $\hat{F}$, numerical values of its components do not depend on coordinate system. That is, for example, the component $\hat{F}_{11}$ or $\bar{F}_{1}$ is $-i B_{3}$. If we use another coordinate system $\left\{x^{\prime \mu}\right\}$, matrices of reference vectors are changed to $\left\{\hat{\gamma}^{\prime \mu}\right\}$ but it is kept that $\hat{F}_{11}=\bar{F}_{1}=-i B_{3}$, where $B_{3}$ is the third component of the magnetic field vector in the coordinate system $\left\{x^{\mu}\right\}$. Thus the column $\bar{F}$ in (2.18) is also an invariant object.

In this connection we should recall [8] in which Dirac introduces his equation. In this article he considers the case when the unknown column is invariant. On the other hand, Pauli [13] has proposed to keep the $\gamma$-matrices invariable when the coordinates are transformed. In this case, we must transform the unknown column into Dirac equation. In our designations this means that

$$
\gamma^{\mu} \frac{\partial \bar{F}}{\partial x^{\mu}}=0 \longrightarrow \gamma^{\mu} \frac{\partial \bar{F}^{\prime}}{\partial x^{\prime \mu}}=0 \Longrightarrow \bar{F}^{\prime}=\hat{\Lambda} \bar{F}
$$

where it is essential that $\hat{\Lambda} \neq \hat{\Lambda}(x)$.

This transformation for column $\bar{F}$ is called spinor transformation. On this topic, see [15], where comparative analysis for these two approaches to the invariance of Dirac equation with respect to Lorentz transformations is given.

Though in (3.4) we have Lorentz transformation for coordinates (3.2), transformation (3.4) is not space-time rotation for Maxwell equations in massless Dirac equation form (2.18). Really, so far as we keep the relation between components of $\bar{F}$ and $E_{i}$, $B_{i}$ (2.16), transformation (3.4) changes the vector components $E_{i}, B_{i}$ to components 
of some other vector $E_{i}^{\prime}, B_{i}^{\prime}$ which, in general, may be complex numbers. But transformation (3.4) keeps (2.18) invariant. Hence, this transformation gives new solution of (2.18)

$$
\bar{F}\left(x^{\mu}\right) \longrightarrow \hat{\Lambda} \bar{F}\left(L_{. v}^{\mu} x^{v}\right) .
$$

Here the matrices $\hat{\Lambda}$ and $\left(L_{. v}^{\mu}\right)$ are interconsistent in the sense of the relation $L_{. \mu}^{v} \hat{\gamma}^{\mu}=$ $\hat{\Lambda}^{-1} \hat{\gamma}^{v} \hat{\Lambda}$, but transformation (3.5) is not real space-time rotation. In particular, if Lorentz matrix $\left(L_{. v}^{\mu}\right)$ corresponds to the space rotation for $2 \pi$, then transformation (3.5) changes any solution of Maxwell equations to the same solution with opposite sign.

Equation (2.18) may be called spinor representation for Maxwell equations.

4. Born-Infeld electrodynamics and Clifford numbers. Equations of Born-Infeld electrodynamics in arbitrary coordinates (and outside of singularities) are written in the following form (see [4]):

$$
\begin{gathered}
\sqrt{|g|}^{-1} \partial_{i} \sqrt{|g|} D^{i}=0, \\
\sqrt{|g|}^{-1} \partial_{i} \sqrt{|g|} B^{i}=0, \\
\sqrt{|g|}^{-1} \partial_{0} \sqrt{|g|} D^{i}+\varepsilon^{0 i j k} \partial_{j} H_{k}=0, \\
\sqrt{|g|}^{-1} \partial_{0} \sqrt{|g|} B^{i}-\varepsilon^{0 i j k} \partial_{j} E_{k}=0,
\end{gathered}
$$

where $E_{i} \equiv F_{i 0}, B^{i} \equiv-(1 / 2) \varepsilon^{0 i j k} F_{j k}, D^{i} \equiv f^{0 i}, H_{i} \equiv(1 / 2) \varepsilon_{0 i j k} f^{j k}$, and

$$
\begin{aligned}
& f^{\mu \nu}=\frac{1}{\mathscr{L}}\left[F^{\mu \nu}-\frac{\chi^{2}}{2} g \varepsilon^{\mu \nu \sigma \rho} F_{\sigma \rho}\right], \\
& \mathscr{L} \equiv \sqrt{\left|1-\chi^{2} \mathscr{I}-\chi^{4} \mathscr{g}^{2}\right|}, \quad \mathscr{I} \equiv \frac{1}{2} F_{\mu \nu} F^{\nu \mu}, \mathscr{g} \equiv \frac{1}{8} \varepsilon_{\mu \nu \sigma \rho} F^{\mu \nu} F^{\sigma \rho},
\end{aligned}
$$

where $x$ is some dimensional constant.

By analogy with (2.1) and (2.9), we have

$$
\hat{f}=\frac{1}{2} f^{\mu \nu} \hat{\gamma}_{\mu} \hat{\gamma}_{v}=\hat{D}-\hat{\imath} \hat{H}
$$

Now consider the following equation:

$$
\hat{\partial} \cdot \hat{F}+\hat{\partial} \wedge \hat{f}=0
$$

By analogy with derivation from (2.11) to (2.13), we have from (4.5) that

$$
\begin{gathered}
\hat{\gamma}^{i} \partial_{i} \wedge \hat{D}=0, \\
\hat{\gamma}^{i} \partial_{i} \cdot \hat{\imath} \hat{B}=0, \\
\hat{\gamma}^{0} \partial_{0} \wedge \hat{D}-\hat{\gamma}^{i} \partial_{i} \wedge \hat{\imath} \hat{H}=0, \\
\hat{\gamma}^{0} \partial_{0} \cdot \hat{\imath} \hat{B}-\hat{\gamma}^{i} \partial_{i} \cdot \hat{E}=0 .
\end{gathered}
$$


This system conforms with system (4.1) in the coordinates for which $\hat{\gamma}^{\mu} \neq \hat{\gamma}^{\mu}(x)$. But coordinate-free equation (4.5) fully conforms with system (4.1) for any coordinates. This is also verified by direct substitution.

As we can see, system (4.1) is appropriate for any nonlinear electrodynamics model. Thus, (4.5) is a Clifford number form for equations of nonlinear electrodynamics. In this case the relation between $\hat{f}$ and $\hat{F}$ defines concrete model. It can easily be shown that Born-Infeld relation (4.2) is written in the form

$$
\begin{gathered}
\hat{f}=\frac{1}{\mathscr{L}}\left(\hat{\imath}+\hat{\imath} \chi^{2} \mathscr{f}\right) \hat{F}, \\
\mathscr{I} \hat{1}=\frac{1}{2}\left[\hat{F}^{2}+\left(\hat{F}^{\dagger}\right)^{2}\right], \quad \mathscr{L} \hat{1}=\frac{1}{4} \hat{\imath}\left[\hat{F}^{2}-\left(\hat{F}^{\dagger}\right)^{2}\right] .
\end{gathered}
$$

Making elementary transformations with (4.5) and extracting the first column in matrix equation (see (2.16) and (2.18)), we obtain

$$
\hat{\partial} \bar{F}=[\hat{\partial} \wedge(\hat{F}-\hat{f})] \overline{1} .
$$

Using relation of type (4.7) and representation (2.17), the right-hand side of (4.9) can be represented by $\bar{F}$ and $\bar{F}^{*}$. Thus, we have the nonlinear like Dirac equation which is the form of nonlinear electrodynamics equations.

5. Conclusion. We have considered the Clifford number formalism for Maxwell equations. The Clifford imaginary unit for space-time $\hat{\imath}$ is introduced as coordinate independent form of fully antisymmetric fourth-rank tensor. Earlier (see [12]) the pseudoscalar $\hat{\gamma}^{0} \hat{\gamma}^{1} \hat{\gamma}^{2} \hat{\gamma}^{3}$ was used as such imaginary unit. This distinction plays an important role for curvilinear coordinates and for generalization to curved spacetime.

We have considered the representation of Maxwell equations in massless Dirac equation form. In this connection, we have discussed two approaches to the invariance of Dirac equation with respect to the Lorentz transformations. According to the first unjustly forgotten approach, the unknown column is invariant and according to the second approach, it has the transformation properties known as spinorial ones.

We have obtained the Clifford number representation for nonlinear electrodynamics equations. From this representation, we obtain the nonlinear like Dirac equation which is the form of nonlinear electrodynamics equations. As a special case, we have obtained the appropriate representations for Born-Infeld nonlinear electrodynamics.

These representations can help to obtain new solutions in Born-Infeld electrodynamics. Also such approach may make clear the relations between nonlinear electrodynamics and particle physics.

\section{REFERENCES}

[1] M. Born and L. Infeld, Foundations of the new field theory, Proc. Roy. Soc. London Ser. A 144 (1934), 425-451.

[2] N. A. Chernikov and N. S. Shavokhina, The Born-Infeld theory as part of Einstein's unified field theory, Soviet Math. (Iz. VUZ) 30 (1986), 81-83.

[3] A. A. Chernitskii, Light beams distortion in nonlinear electrodynamics, J. High Energy Phys. 1998 (1998), no. 11, Paper 15, 1-5. 
[4] _ Nonlinear electrodynamics with singularities (modernized Born-Infeld electrodynamics), Helv. Phys. Acta 71 (1998), no. 3, 274-287.

[5]___ Dyons and interactions in nonlinear (Born-Infeld) electrodynamics, J. High Energy Phys. 1999 (1999), no. 12, Paper 10, 1-34.

[6] V. I. Denisov, New effect in nonlinear Born-Infeld electrodynamics, Phys. Rev. D 61 (2000), no. 3, 036004, 1-7.

[7] S. Deser, J. McCarthy, and Ó. Sarioglug̃lu, "Good propagation" constraints on dual invariant actions in electrodynamics and on massless fields, Classical Quantum Gravity 16 (1999), 841-847.

[8] P. A. M. Dirac, The quantum theory of the electron, Proc. Roy. Soc. Ser. A 117 (1928), 610-624.

[9] A. S. Eddington, The Mathematical Theory of Relativity, Cambridge University Press, Cambridge, 1924.

[10] A. Einstein and B. Kaufman, A new form of the general relativistic field equations, Ann. of Math. (2) 62 (1955), 128-138.

[11] E. S. Fradkin and A. A. Tseytlin, Nonlinear electrodynamics from quantized strings, Phys. Lett. B 163 (1985), no. 1-4, 123-130.

[12] D. Hestenes and G. Sobczyk, Clifford Algebra to Geometric Calculus. A Unified Language for Mathematics and Physics, Fundamental Theories of Physics, vol. 18, D. Reidel Publishing, Dordrecht, 1984.

[13] W. Pauli, Contributions mathématique à la théorie des matrices de Dirac, Ann. Inst. H. Poincaré 6 (1936), 109-136 (French).

[14] M. Riesz, Clifford Numbers and Spinors, Fundamental Theories of Physics, vol. 54, Kluwer Academic Publishers, Dordrecht, 1993.

[15] A. Sommerfeld, Atombau und Spektrallinien, II, Friedr, Vieweg \& Sohn, Braunschweig, 1951 (German).

Alexander A. Chernitskit: Saint Petersburg Electrotechnical University, Prof. POPOV STREET 5, ST. PETERSBURG 197376, RUSSIA

E-mail address: aa@cher.etu.spb.ru 


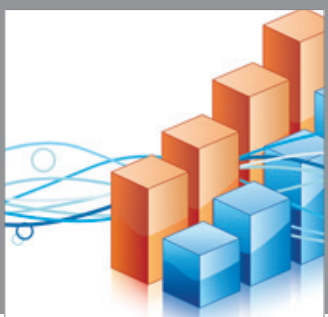

Advances in

Operations Research

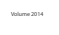

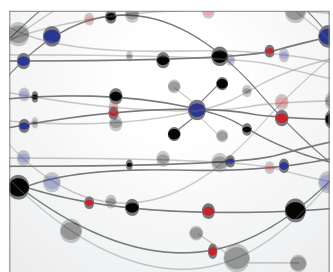

\section{The Scientific} World Journal
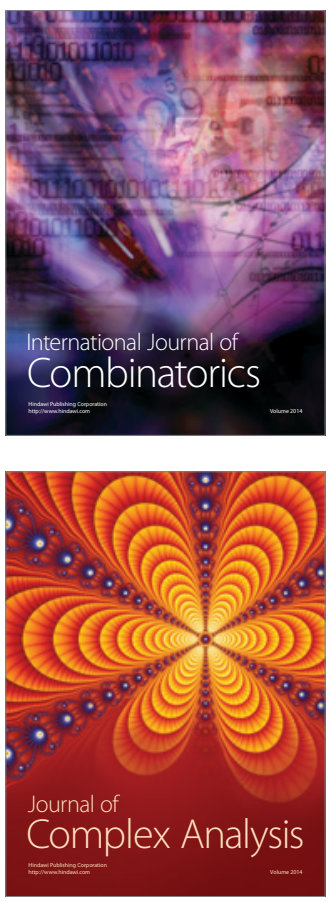

International Journal of

Mathematics and

Mathematical

Sciences
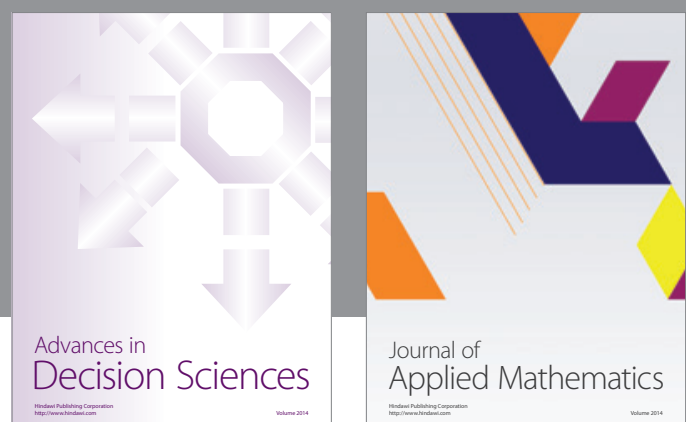

Journal of

Applied Mathematics
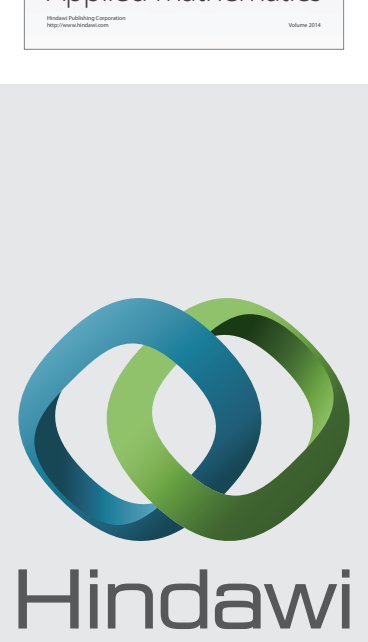

Submit your manuscripts at http://www.hindawi.com
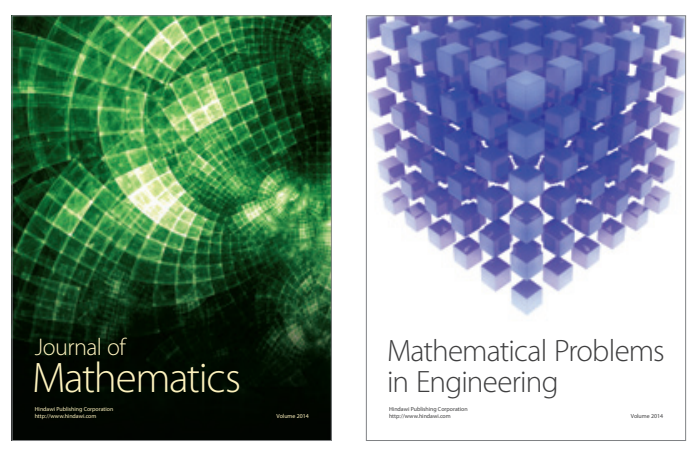

Mathematical Problems in Engineering
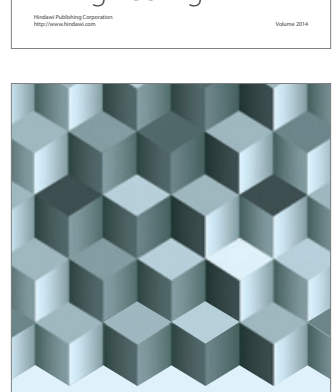

Journal of

Function Spaces
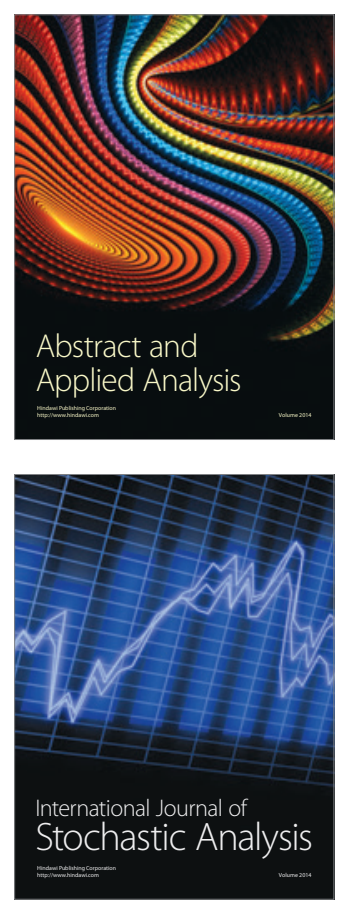

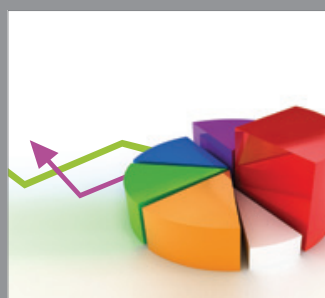

ournal of

Probability and Statistics

Promensencen
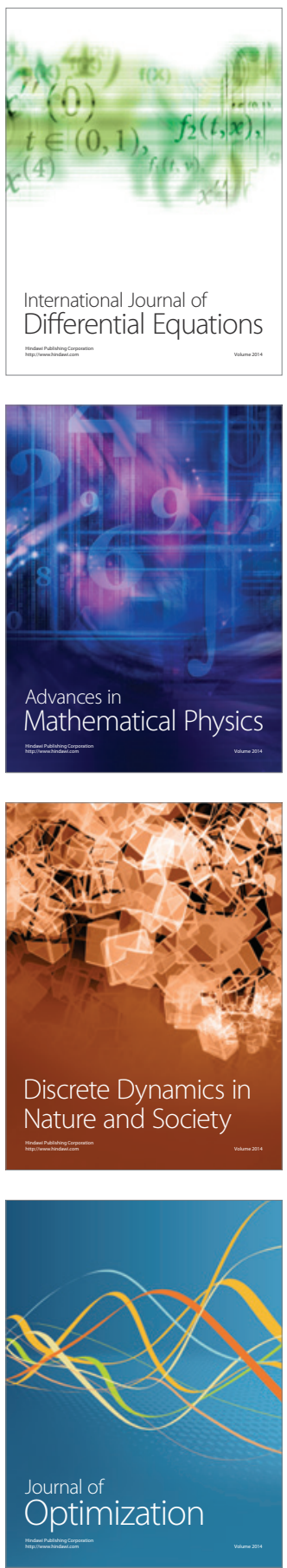